\title{
Modeling Urgency in Component-Based Real-time Systems
}

\author{
Nguyen Van Tang ${ }^{1}$, Dang Van Hung ${ }^{2}$, Mizuhito Ogawa ${ }^{1}$ \\ 1 Japan Advanced Institute of Science and Technology \\ E-mail: \{tang_nguyen,mizuhito\}@jaist.ac.jp \\ 2 United Nation University, International Institute for Software Technology \\ E-mail: dvh@iist.unu.edu
}

\begin{abstract}
A component-based realtime system is a simple model for the server-client relation with time constraints. This paper presents an efficient algorithm, called a blackbox testing algorithm, for detecting the emptiness of a component-based realtime system. This algorithm was originally proposed in [5], but with a certain flaw. We improve it and correct the flaw by using urgency [2] of transitions.
\end{abstract}

Keywords: Component Software, Duration Automata, Automatic Verification, Real-time Systems, Model Checking.

\section{Introduction}

The architectural design for embedded systems often relies on specification of the interface of components only, without accessing their internal behaviors. Based on this observation, a simple model for component-based real-time systems based on duration automata was proposed in [5]. A duration automaton does not have clock variables like a time automaton [1], but simply has an upper bound and a lower bound for each transition. A component-based real-time system is defined as a system consisting of a host, which is a general duration automaton, and several components which are duration automata with certain restrictions. A component-based real-time system can be regarded as a timed automaton, thus its emptiness is PSPACE-complete.

This paper presents an efficient algorithm for detecting the emptiness, called a blackbox testing algorithm. This algorithm was originally proposed in [5], but with certain flaws. We improve it and correct these flaws by using urgency of transitions, which was firstly introduced by Bornot et. al. [2] as a technique for choosing time deadline condition in complex system specifications.

\section{Duration Automata}

Duration automata was firstly introduced in [3] for modeling simple real-time systems. A duration automaton is a finite automaton in which each transition must occur in an associated time interval. Let $\mathbb{R}^{+}$be the set of non-negative real numbers, and let Intv $=\left\{[l, u] \mid l \in \mathbb{R}^{+}, u \in \mathbb{R}^{+} \cup\{\infty\}\right\}$. 
Definition 1. A duration automaton is a tuple $M=\langle S, \tilde{\Sigma}, q, R, F\rangle$, where

1. $S$ is a finite set of states,

2. $\tilde{\Sigma}$ is alphabet of actions,

3. $q \in S$ is the initial state,

4. $R \subseteq S \times \tilde{\Sigma} \times I n t v \times S$ is timed transition relation, and

5. $F \subseteq S$ is the set of final states.

Each element of $M$ is referred by $S(M), \tilde{\Sigma}(M), R(M), q(M)$, and $F(M)$, respectively. An untimed automaton untimed $(M)$ is obtained by forgetting time constraints, i.e., replacing $R$ with untimed $(R)=\left\{\left(s, a, s^{\prime}\right) \mid\left(s, a,[l, u], s^{\prime}\right) \in R\right\}$. As in standard terminology,

- A configuration of $M$ is a pair $(s, d) \in S \times \mathbb{R}^{+}$.

- The initial configuration of $M$ is $(q, 0)$.

- An acceptance configuration of $M$ is a configuration $(s, d)$ where $s \in F$.

A duration automaton is equivalent to a timed automata with a single clock such that each transition resets it. A configuration $(s, d)$ is regarded as a state $s$ with a clock $d$.

- A transition of $M$ on configurations is either a time transition $(s, d) \stackrel{\delta}{\rightarrow}$ $(s, d+\delta)$ or a discrete transition $(s, d) \stackrel{\delta}{\rightarrow} \stackrel{\mathrm{a}}{\rightarrow}\left(s^{\prime}, 0\right)$ where $a \in \tilde{\Sigma}, \delta \geq 0$, $l \leq d+\delta \leq u$, and $\left(s, a,[l, u], s^{\prime}\right) \in R$.

- A (possibly empty) sequence $w=\left(a_{1}, t_{1}\right) \ldots\left(a_{k}, t_{k}\right) \in\left(\tilde{\Sigma} \times \mathbb{R}^{+}\right)^{*}$ is a timed word of $M$ if and only if there is a run $\left(s_{0}, 0\right) \stackrel{\delta_{1}}{\longrightarrow} \stackrel{a_{1}}{\longrightarrow}\left(s_{1}, 0\right) \stackrel{\delta_{2}}{\longrightarrow} \stackrel{a_{2}}{\longrightarrow} \ldots \stackrel{\delta_{k}}{\longrightarrow} \stackrel{a_{k}}{\longrightarrow}$ $\left(s_{k}, 0\right)$ such that $s_{0}=q, s_{k} \in F, t_{1}=\delta_{1}$ and $t_{i+1}-t_{i}=\delta_{i+1}$ for $1 \leq i \leq k-1$.

Theorem 1. Duration automata is closed under union, intersection and complementation. Decision problems for duration automata are decidable.

Proof. (Sketch) For a given duration automaton $M$, one can reduce $M$ to a finite automaton $M^{\prime}$. We first list the endpoints of intervals (lower and upper bounds of intervals) of transitions in $M$ as an increasing sequence, say, $0=p_{0}<p_{1}<p_{2} \ldots<p_{n}<\infty$. This is possible because the number of transitions of $M$ is finite. Secondly, we define the set of basic intervals $B I=$ $\left\{\left[p_{0}, p_{1}\right], \ldots,\left[p_{n-1}, p_{n}\right],\left[p_{n}, \infty\right)\right\}$. Since each interval appeared in a transition of $M$ is the union of certain basic intervals. So, each transition of $M$ can be divided into several ones. For instance, $\left(s, a,\left[p_{0}, p_{2}\right], s^{\prime}\right)$ can be divided into $\left(s, a,\left[p_{0}, p_{1}\right], s^{\prime}\right)$ and $\left(s, a,\left[p_{1}, p_{2}\right], s^{\prime}\right)$. We now construct a finite automaton $M^{\prime}$ such that $S\left(M^{\prime}\right)=S(M), F\left(M^{\prime}\right)=F(M)$, the input alphabet of $M^{\prime}$ is $\tilde{\Sigma}\left(M^{\prime}\right)=$ $\tilde{\Sigma}(M) \times B I$. Let $\left(s,\left(a,\left[p_{i}, p_{i+1}\right]\right), s^{\prime}\right) \in R\left(M^{\prime}\right)$ if $\left(s, a,\left[p_{i}, p_{i+1}\right], s^{\prime}\right) \in R(M)$. Clearly, $M^{\prime}$ accepts a word $\left(a_{1},\left[l_{1}, u_{1}\right]\right) \ldots\left(a_{n},\left[l_{n}, u_{n}\right]\right)$ if and only if $M$ accepts the timed word $\left(a_{1}, t_{1}\right) \ldots\left(a_{n}, t_{n}\right)$, where $t_{0}=0$ and $\left(l_{i} \leq t_{i}-t_{i-1} \leq u_{i}\right)$ for $1 \leq i \leq n$. Thus, the emptiness and the closure properties of duration automata are reduced to that of finite automata, respectively. 


\section{Synchronized Composition Systems}

Duration interface automata is duration automata in which the input alphabet $\tilde{\Sigma}$ is decomposed into pairwise disjoint alphabets $\Sigma, \Delta$ and $\nabla$, which correspond to internal, input and output actions, respectively.

Definition 2. A host is a duration interface automaton. A component is a duration interface automaton $X=\langle S, \Sigma \cup \Delta \cup \nabla, q, R, F\rangle$ that satisfies:

$-\Sigma=\emptyset$ (i.e., no "explicit" internal actions).

- $\left(s, a,[l, u], s^{\prime}\right) \in R \wedge a \in \Delta$ implies $l=0 \wedge u=\infty$ (i.e., an input can occur anytime).

- $\left(s, a,[l, u], s^{\prime}\right) \in R \wedge a \in \nabla$ implies $u=\infty$ (i.e., when an output is ready, it can be sent at any time afterward).

Definition 3. A synchronized composition system $S y s=\left\langle M, X_{1}, \cdots, X_{k}\right\rangle$ consists of a single host $M$ and components $X_{1}, \cdots, X_{k}$ such that $\tilde{\Sigma}\left(X_{i}\right) \cap \tilde{\Sigma}\left(X_{j}\right)=\emptyset$ for each $i \neq j, \Sigma(M) \cap \tilde{\Sigma}\left(X_{i}\right)=\emptyset$ for each $i, \Delta(M)=\bigcup_{i=1}^{k} \nabla\left(X_{k}\right), \nabla(M)=$ $\bigcup_{i=1}^{k} \Delta\left(X_{k}\right)$, and

- The set of configurations is $\left\{\left(\left(s_{0}, d_{0}\right),\left(s_{1}, d_{1}\right), \cdots,\left(s_{k}, d_{k}\right) \mid s_{0} \in S(M), s_{1} \in\right.\right.$ $\left.S\left(X_{1}\right), \cdots, s_{k} \in S\left(X_{k}\right), d_{i} \in \mathbb{R}^{+}\right\}$.

- A transition is $\left(\left(s_{0}, d_{0}\right),\left(s_{1}, d_{1}\right), . .,\left(s_{k}, d_{k}\right)\right) \stackrel{\delta}{\rightarrow} \stackrel{a}{\longrightarrow}\left(\left(s_{0}^{\prime}, d_{0}^{\prime}\right),\left(s_{1}^{\prime}, d_{1}^{\prime}\right), . .,\left(s_{k}^{\prime}, d_{k}^{\prime}\right)\right)$ for $\delta \geq 0$ and $a \in \bigcup_{i=1}^{k} \tilde{\Sigma}\left(X_{i}\right)$, if there exists $i$ with $1 \leq i \leq k$ such that

- $a \in \tilde{\Sigma}\left(X_{i}\right)$,

- $l_{0} \leq d_{0}+\delta \leq u_{0}$ and $l_{i} \leq d_{i}+\delta \leq u_{i}$ (called synchronization condition) for $\left(s_{i}, a,\left[l_{i}, u_{i}\right], s_{i}^{\prime}\right) \in R\left(X_{i}\right)$ and $\left(s_{0}, a,\left[l_{0}, u_{0}\right], s_{0}^{\prime}\right) \in R(M)$,

- $d_{0}^{\prime}=d_{i}^{\prime}=0$, and

- $\left(s_{j}^{\prime}, d_{j}^{\prime}\right)=\left(s_{j}, d_{j}+\delta\right)$ for $j \neq 0, i$.

- A run is a sequence of transitions that starts from the initial configuration $\left((q(M), 0),\left(q\left(X_{1}\right), 0\right), \cdots,\left(q\left(x_{k}\right), 0\right)\right)$.

- A timed word $\left(a_{1}, t_{1}\right) \cdots\left(a_{k}, t_{k}\right)$ with $t_{1}=\delta_{1}$ and $t_{i+1}=t_{i}+\delta_{i+1}$ is accepted if there is a run $\left((q(M), 0),\left(q\left(X_{1}\right), 0\right), \cdots,\left(q\left(x_{k}\right), 0\right)\right) \stackrel{\delta_{1}}{\longrightarrow} \stackrel{a_{1}}{\longrightarrow} \cdots \stackrel{\delta_{k}}{\longrightarrow} \underset{a_{k}}{\longrightarrow}$ $\left(\left(s_{0}, d_{0}\right),\left(s_{1}, d_{1}\right), . .,\left(s_{k}, d_{k}\right)\right)$ with $s_{0} \in F(M), s_{1} \in F\left(X_{1}\right), \cdots, s_{k} \in F\left(X_{k}\right)$.

Theorem 2. A synchronized composition system Sys $=\left\langle M, X_{1}, \cdots, X_{k}\right\rangle$ is a timed automaton with $k+1$ clocks such that each transition with a time constraint $l_{i} \leq d_{i} \leq u_{i}$ on a clock $d_{i}$ will reset $d_{i}$ to 0 .

Proof. (Sketch) Let $\mathcal{C}$ be the set of time constraints $\left[l_{j}, u_{j}\right]$ appearing in a host $M$ and components $X_{i}$. Note that $l_{j}, u_{j} \in \mathbb{R}^{+}$. Assume that we can choose $\mathcal{C}^{\prime}$ (a digitization of $\mathbb{C}$ ) consisting of rational time constraints $\left[l_{j}^{\prime}, u_{j}^{\prime}\right]$ such that there is a run of Sys if and only if there is a run of $S y s^{\prime}$, where $S y s^{\prime}$ is obtained replacing each $\left[l_{j}, u_{j}\right]$ with its digitization $\left[l_{j}^{\prime}, u_{j}^{\prime}\right]$. Then, the proof has done.

Let $\operatorname{rat}(\mathcal{C})$ be the set of rational numbers appearing in $\mathcal{C}$ and let $m$ be a common multiplier of dominators of positive elements in $\operatorname{rat}(\mathcal{C})$. Let $\operatorname{irr}(\mathcal{C})$ be the set of irrational numbers appearing in $\mathcal{C}$ and let $\operatorname{lin}(\mathcal{C})$ be the set of all possible 
linear combinations of $\operatorname{irr}(\mathcal{C})$ with natural numbers (i.e., $\operatorname{lin}(\mathcal{C})=\left\{n_{1} \alpha_{1}+\cdots+\right.$ $\left.\left.n_{l} \alpha_{l} \mid n_{j} \in \mathbb{N}, \alpha_{j} \in \operatorname{irr}(\mathcal{C})\right\}\right)$. Assume that $(\alpha, \beta)$ is the pair such that $\alpha \in \operatorname{irr}(\mathcal{C})$, $\beta \in \operatorname{lin}(\mathcal{C})$, and $\epsilon_{\alpha, \beta}=\frac{\alpha}{\beta}-\left[\frac{\alpha}{\beta}\right]>0$. Since a pair $(\alpha, \beta)$ with $\alpha \in \operatorname{irr}(\mathcal{C})$, $\beta \in \operatorname{lin}(\mathcal{C})$, and $\beta<\alpha$ is finitely many, $(\alpha, \beta)$ with $\epsilon_{\alpha, \beta}$ to be the least exists. We choose a sufficient large multiplier $\bar{m}$ of $m$ such that $\frac{1}{\bar{m}}<\min \left(\frac{\epsilon_{\alpha, \beta}}{2}, \frac{1-\epsilon_{\alpha, \beta}}{2}\right)$, and set $l_{j}^{\prime}=\frac{\left[\bar{m} l_{j}\right]}{\bar{m}}$ and $u_{j}^{\prime}=\frac{\left[\bar{m} u_{j}\right]}{\bar{m}}$ for each $l_{j}, u_{j} \in \mathbb{C}$.

Example 1. Fig. 3 shows a simple synchronized composition system $S y s=\left\langle X_{1}, X_{2}\right\rangle$ and its corresponding timed automaton $\mathcal{A}$.

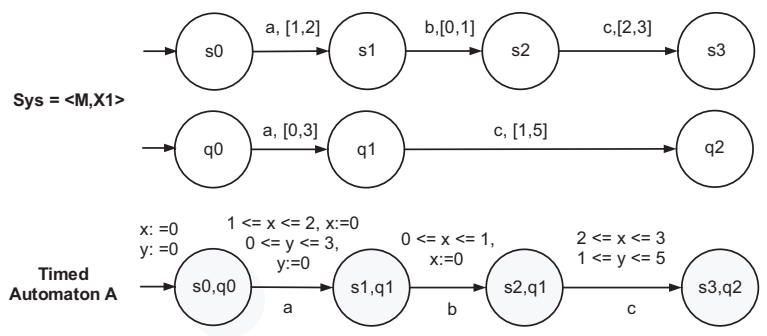

Fig. 1. Synchronized Composition System as a Timed Automaton

From Theorem 2, the emptiness problem of a component-based realtime system is decidable. However, its complexity is expensive, i.e., PSPACE-complete [1] after digitization of time constraints.

\section{Component-based realtime systems}

Definition 4. A component $X$ is input/output deterministic if

- for $a \in \Delta(X),\left(s, a,[0, \infty), s^{\prime}\right),\left(s, a,[0, \infty), s^{\prime \prime}\right) \in R(X)$ implies $s^{\prime \prime}=s^{\prime} \quad$ (input determinism), and

- for $b \in \nabla(X)$ and $b^{\prime} \in \nabla(X) \cup \Delta(X),\left(s, b,[l, \infty), s^{\prime}\right),\left(s, b^{\prime},\left[l^{\prime}, u^{\prime}\right], s^{\prime \prime}\right) \in R(X)$ implies $s^{\prime \prime}=s^{\prime}, l^{\prime}=l, u^{\prime}=\infty$, and $b^{\prime}=b$ (output determinism).

A synchronized composition system Sys $=\left\langle M, X_{1}, \cdots, X_{k}\right\rangle$ is a componentbased realtime system [5] if each component $X_{i}$ is input/output deterministic.

Definition 5. We borrow notations from Definition 3. In a component-based system Sys $=\left\langle M, X_{1}, \cdots, X_{k}\right\rangle$, a transition $\left(\left(s_{0}, d_{0}\right),\left(s_{1}, d_{1}\right), . .,\left(s_{k}, d_{k}\right)\right) \stackrel{\delta}{\rightarrow} \stackrel{a}{\rightarrow}$ $\left(\left(s_{0}^{\prime}, d_{0}^{\prime}\right),\left(s_{1}^{\prime}, d_{1}^{\prime}\right), . .,\left(s_{k}^{\prime}, d_{k}^{\prime}\right)\right)$ is urgent if $\delta$ is the minimum among synchronization conditions of all possible transitions from $\left(\left(s_{0}, d_{0}\right),\left(s_{1}, d_{1}\right), . .,\left(s_{k}, d_{k}\right)\right)$, and delayable otherwise. We also say a corresponding transition $\left(s_{0}, a,\left[l_{0}, u_{0}\right], s_{0}^{\prime}\right) \in$ $R(M)$ of a host is urgent, and delayable otherwise. 
Definition 6. Let $w=\left(a_{1}, t_{1}\right) \cdots\left(a_{k}, t_{k}\right)$ and let $a_{i} \in A$. For $B \subseteq A$, the projection $\left.w\right|_{B}$ is the subsequence of $w$ obtained by filtering each element $\left(a_{j}, t_{j}\right)$ with $a_{j} \in B$. For $a_{j} \in B,\left(a_{h}, t_{h}\right)$ is a local predecessor of $\left(a_{j}, t_{j}\right)$ wrt $B$, if $a_{h} \in B, h<j$, and $a_{i} \notin B$ for each $i$ with $h<i<j$.

Definition 7. Let Sys $=\left\langle M, X_{1}, \cdots, X_{k}\right\rangle$ be a component-based real-time system. For a timed word $w=\left(a_{1}, t_{1}\right) \ldots\left(a_{n}, t_{n}\right)$, let $a_{j} \in \nabla\left(X_{i}\right)$ and let $\left(a_{h}, t_{h}\right)$ be the local predecessor of $\left(a_{j}, t_{j}\right)$ wrt $\tilde{\Sigma}\left(X_{i}\right)$. For $\left(s^{\prime}, a_{j},\left[d_{j}, \infty\right), s^{\prime \prime}\right) \in R\left(X_{i}\right)$ with $q\left(X_{i}\right) \stackrel{\text { untime }\left(\left.w\right|_{\tilde{\Sigma}\left(X_{i}\right)}\right)}{\longrightarrow} s^{\prime}$ in untimed $\left(X_{i}\right), d_{j}$ is the minimum delay at $\left(a_{j}, t_{j}\right)$.

Definition 8. A consecutive sequence of transitions $\left(s_{i-1}, a_{i},\left[l_{i}, u_{i}\right], s_{i}\right) \in R(M)$ $(i=1, \cdots, n)$ is called an accepted sequence of transitions of the host $M$ if $s_{0}=q(M)$ and $s_{n} \in F(M)$.

Note that such a minimum delay is well-defined, since each component in Sys is input/output deterministic. Let $r$ be the number of states of $M$, and let $m$ is the maximal number of states of components $X_{j}, j \leq k$. Let $P$ be the length of the longest path (number of transitions) from the initial state to a final state of $M$ in which any cycle is not repeated more than $r * m^{k}$ times. The next theorem reduces the emptiness of a whole component-base realtime system to that of its host under certain conditions.

Theorem 3. Let Sys $=\left\langle M, X_{1}, \cdots, X_{k}\right\rangle$ be a component-based realtime system. There is an accepted timed word of Sys if and only if there are an accepted sequence of transitions of the host $M \sigma=\left(s_{0}, a_{1},\left[l_{1}, u_{1}\right], s_{1}\right)\left(s_{1}, a_{2},\left[l_{2}, u_{2}\right], s_{3}\right) \ldots$ $\left(s_{n-1}, a_{n},\left[l_{n}, u_{n}\right], s_{n}\right)$ with the length $n \leq P$, and a real number sequence $0=$ $t_{0} \leq t_{1} \leq \cdots \leq t_{n}$ satisfying following conditions:

$-w_{i}=\left.a_{1} a_{2} \ldots a_{n}\right|_{\tilde{\Sigma}\left(X_{i}\right)}$ is accepted by untimed $\left(X_{i}\right)$ for each $i$ with $1 \leq i \leq k$, $-l_{i} \leq t_{i}-t_{i-1} \leq u_{i}$ for all $i$ with $1 \leq i \leq n$,

- When $a_{j} \in \nabla\left(X_{i}\right)$, let $\left(a_{h}, t_{h}\right)$ be the local predecessor of $\left(a_{j}, t_{j}\right)$ wrt $\tilde{\Sigma}\left(X_{i}\right)$ and let $d_{j}$ be the minimum delay at $\left(a_{j}, t_{j}\right)$. Then,

- $t_{j}-t_{h} \geq d_{j}$, and

- if a transition $\left(s_{j}, 0\right) \stackrel{\delta}{\rightarrow} \stackrel{a_{j}}{\longrightarrow}\left(s_{j+1}, 0\right)$ is urgent, $t_{j}=\min \left\{t \mid t-t_{h} \geq\right.$ $\left.d_{j} \wedge l_{j} \leq t-t_{j-1} \leq u_{j}\right\}$.

Proof. (Sketch) We only have to prove the bound $P$ in "only if" part. Assume that a timed word $w=\left(a_{1}, t_{1}\right) \cdots\left(a_{n}, t_{n}\right)$ is accepted by Sys. Timed word $w$ is inductively computed by constructing an accepted sequence of transitions $\phi=\left(s_{0}, a_{1},\left[l_{1}, u_{1}\right], s_{1}\right)\left(s_{1}, a_{2},\left[l_{2}, u_{2}\right], s_{3}\right) \cdots\left(s_{n-1}, a_{n},\left[l_{n}, u_{n}\right], s_{n}\right)$ of the host $M$. If $n \leq P$, the proof is done. If $n>P$, then $\phi$ must include at least a cycle $c$ with more than $r * m^{k}$ repetitions. By the pumping lemma like argument, we can find a shorter accepted sequence of transitions of $M$ that satisfies all the conditions in the Theorem.

In the next section, the blackbox testing algorithm will be presented by searching an accepted sequence of the host $M$ satisfying the conditions in Theorem 3 up to the length $P$. 


\section{Checking Emptiness of Component-based Realtime Systems}

The emptiness problem for a system plays a key role in checking the safety. An algorithm for checking the emptiness of a component-based system using black box testing was originally proposed in [5]. However, there is a flaw such that a component-based realtime system is empty, whereas the algorithm in [5] reports that the system is not empty. For instance, consider the following simple example.

Example 2. Let Sys $=\langle M, X\rangle$ where $M$ is a host and $X$ is a component.

- $M=\left\langle\left\{s_{0}, s_{1}, s_{1}^{\prime}\right\},\{a\}, s_{0},\left\{\left(s_{0}, a,[2,4], s_{1}\right),\left(s_{0}, a,[5,10], s_{1}^{\prime}\right)\right\},\left\{s_{1}^{\prime}\right\}\right\rangle$.

- $X=\left\langle\left\{q_{0}, q_{1}\right\},\{a\}, q_{0},\left\{\left(q_{0}, a,[3, \infty), q_{1}\right)\right\},\left\{q_{1}\right\}\right\rangle$.

In [5], the state $\left(s_{1}^{\prime}, q_{1}\right)$ is regarded as a successor of $\left(s_{0}, q_{0}\right)$. But, $\left(s_{1}^{\prime}, q_{1}\right)$ is not reachable from $\left(s_{0}, q_{0}\right)$. This is due to the fact that Sys has already changed from $\left(s_{0}, q_{0}\right)$ to $\left(s_{1}, q_{1}\right)$ at some point in the time interval $[3,4]$.

To deal with this problem, we introduce urgency for transitions to specify time deadline condition of configurations. For the emptiness problem, we first use the BlackboxTest algorithm proposed in [5] for solving membership for a component. Secondly, we construct Algorithm 1 to compute time deadline condition of a given configuration. Lastly, with the aid of Algorithm 1 and Theorem 3, we construct Algorithm 2 to check the emptiness of a component-based system using black box testing.

For a sequence of transitions $\phi$, let label $(\phi)$ denote the sequence of the labels corresponding to $\phi$. For a given prefix of a generated sequence of transitions $\sigma=e_{1} e_{2} \ldots e_{n}$, where $e_{i}=\left(s_{i-1}, a_{i},\left[l_{i}, u_{i}\right], s_{i}\right) \in R(M)$ (i =1..n). Suppose that $t_{0}, t_{1}, \ldots, t_{n}$ are inductively computed in advance. Time deadline of $s_{n}$ along $\sigma$ is denoted by deadline ${ }_{\sigma}\left(s_{n}\right)$. It can be computed by the following algorithm:

Algorithm 1. Deadline $_{\sigma}\left(s_{n}\right)$ : (Check the conditions (2) and (3) of Theorem 3)

Input: A prefix-generated sequence $\sigma=e_{1} e_{2} \ldots e_{n}$

Output: deadline $e_{\sigma}\left(s_{n}\right)$.

Method:

1. Compute the set $R\left(s_{n}\right):=\left\{e \mid e=\left(s_{n}, a,[l, u], s\right) \in R(M)\right\}$.

2. deadline $:=\infty$. For $j \leq k$ let $m_{j}$ be the largest index of $\sigma$ such that $a_{m_{j}} \in$ $\tilde{\Sigma}\left(X_{j}\right)$ if it exists, otherwise, set $m_{j}=0$. For each $e=\left(s_{n}, a,[l, u], s\right) \in$ $R\left(s_{n}\right)$.

(a) If $a \in \triangle\left(X_{j}\right) \cup \Sigma(M)$, if BlackboxTest $\left(X_{j},\left.\operatorname{label}(\sigma)\right|_{\tilde{\Sigma}\left(X_{j}\right)}\right)=$ "yes" and $u<$ deadline then deadline $:=u$.

(b) If $a \in \nabla\left(X_{j}\right)$. If BlackboxTest $\left(X_{j}\right.$, label $\left.\left.(\sigma)\right|_{\tilde{\Sigma}\left(X_{j}\right)}\right)=$ "yes", let $d$ be the value of $d_{X_{j}}$. 
- Case 1: $e$ is delayable. If $t_{n}-t_{m_{j}}+u \geq d$ and $u<$ deadline then deadline $:=u$.

- Case 2: $e$ is urgent.

- If $t_{n}-t_{m_{j}}+l \leq d \leq t_{n}-t_{m_{j}}+u$ and $d-\left(t_{n}-t_{m_{j}}\right)<$ deadline then deadline $:=d-\left(t_{n}-t_{m_{j}}\right)$.

- If $t_{n}-t_{m_{j}}+l \geq d$ and $l<$ deadline then deadline $:=l$.

\section{3. return Delainey;}

With the aid of the Algorithm 1, the emptiness of a component-based realtime system can be solved by the following testing procedure.

Algorithm 2. Non-Emptiness(Sys): (Check all conditions of Theorem 3)

Input: Component-based real-time system Sys $=\left\langle M, X_{1}, \cdots, X_{k}\right\rangle$

Output: "Yes" if the set of timed words of Sys is not empty, "No" otherwise. Method:

1. Compute $P$. Generate all accepted sequences of transitions of $M$ with length less than $P$.

2. Check on-the-fly whether any prefix of a generated sequence satisfies the conditions of Theorem 3. This can be done by:

For each prefix of a generated sequence of transitions $\sigma=e_{1} \ldots e_{n-1}$, where $e_{i}=\left(s_{i-1}, a_{i},\left[l_{i}, u_{i}\right], s_{i}\right)$ for each $i$ with $1 \leq i \leq n-1$. Suppose that $t_{0}, t_{1}, \ldots t_{n}$ are inductively computed in advance. For $j \leq k$ let $m_{j}$ be the largest index of $\sigma$ such that $a_{m_{j}} \in \tilde{\Sigma}\left(X_{j}\right)$ if it exists, otherwise, let $m_{j}=0$. For each transition $e_{n}=\left(s_{n-1}, a_{n},[l, u], s\right)$ of the host $M$ starting from $s_{n-1}$. Compute deadline $_{\sigma}\left(s_{n-1}\right)$ using Algorithm 1 . If $l \leq$ deadline $_{\sigma}\left(s_{n-1}\right)$ then:

(a) If $a_{n} \in \triangle\left(X_{j}\right)$, then if: BlackboxTest $\left(X_{j}\right.$, label $\left.\left.(\sigma)\right|_{\tilde{\Sigma}\left(X_{j}\right)}\right)=$ "no", $\sigma e_{n}$ does not satisfy the conditions of Theorem 3. Otherwise, $\sigma:=\sigma e_{n}$, $m_{j}:=n$. If $e_{n}$ is delayable then $t_{n}:=t_{n-1}+u$. If $e_{n}$ is urgent then $t_{n}:=t_{n-1}+l$.

(b) If $a_{n} \in \nabla\left(X_{j}\right)$.

If BlackboxTest $\left(X_{j},\left.\operatorname{label}(\sigma)\right|_{\tilde{\Sigma}\left(X_{j}\right)}\right)=$ "yes", let d be the value of $d_{X_{j}}$. Case 1: If $e_{n}$ is delayable

i. If $t_{n-1}-t_{m_{j}}+u<d$ : then $\sigma e_{n}$ does not satisfy the conditions of Theorem 3 .

ii. If $t_{n-1}-t_{m_{j}}+u \geq d:$ then $\sigma:=\sigma e_{n}, m_{j}:=n, t_{n}:=t_{n-1}+u$.

Case 2: If $e_{n}$ is urgent

i. If $t_{n-1}-t_{m_{j}}+u<d$ then $\sigma e_{n}$ does not satisfy the conditions of Theorem 3 .

ii. If $\left(t_{n-1}-t_{m_{j}}+l\right)<d \leq\left(t_{n-1}-t_{m_{j}}+u\right)$ then the conditions of Theorem 3 are satisfied; update $\sigma:=\sigma e_{n}, t_{n}:=t_{m_{j}}+d, m_{j}:=n$.

iii. If $t_{n-1}-t_{m_{j}}+l \geq d$ then update $\sigma:=\sigma e_{n}, t_{n}:=t_{n-1}+l, m_{j}:=n$. If BlackboxTest $\left(X_{j},\left.\operatorname{label}(\sigma)\right|_{\tilde{\Sigma}\left(X_{j}\right)}\right)=$ "no", the conditions of Theorem 3 are not satisfied.

(c) If $a_{n} \in \Sigma(M)$ then $\sigma:=\sigma e_{n}, t_{n}:=t_{n-1}+u$. 
3. If a generated sequence satisfying the conditions of Theorem 3 is found, return "Yes". Otherwise, return "No".

The complexity for the worst cases of this algorithm is $O\left(P^{2} * K^{P+1}\right)$, where $K=|\tilde{\Sigma}(M)|$ is the size of the alphabet of the system Sys. Unlike the complexity of checking the emptiness for timed automata, this complexity does not depend on the size of the constants occurring in the time intervals for the transitions.

\section{Conclusion}

This paper presented an efficient algorithm for detecting the emptiness, called a blackbox testing algorithm. This algorithm was originally proposed in [5], but with a certain flaw. We improved and correctd it by using urgency of transitions, which was firstly introduced by Bornot et. al. [2] as a technique for choosing time deadline condition in complex system specifications. The urgency enables us to compute the deadline of an accepted behavior of a system using Algorithm 2.

Currently, the algorithm covers checking emptiness only. With the urgency, we can describe a property in Timed Computation Tree Logic (TCTL), such as $\phi \Longrightarrow F_{\leq t} \psi$. The next step is to give an efficent checking algorithm for such TLCL properties of a component-based realtime system.

\section{Acknowledgments}

This research is supported by the 21st Century COE "Verifiable and Evolvable e-Society" funded by Japanese Ministry of Education, Culture, Sports, Science and Technology.

\section{References}

1. R. Alur and D.L. Dill. A Theory of Timed Automata. Theoretical Computer Science, 126: 183-235, 1994.

2. S. Bornot, J. Sifakis, and S. Tripakis. Modeling urgency in timed systems. In COMPOS 1997, 103-129, Springer LNCS 1536, 1997.

3. Zhou Chaochen. Linear Duration Invariants. In Formal Techniques in Real-Time and Fault-Tolerant Systems, pp. 86-109, Springer LNCS 963, 1994.

4. Zhe Dang and Gaoyan Xie. CTL model-checking for systems with unspecified finite state components. In SAVCBS'04, ACM SIGSOFT 2004/FSE-12, pp. 32-38, 2004.

5. Dang Van Hung and Bui Vu Anh. Model Checking Real-time Component Based Systems with Blackbox Testing. In the IEEE proceeding of RTCSA05, page 76-79, Hong Kong, August 2005. 